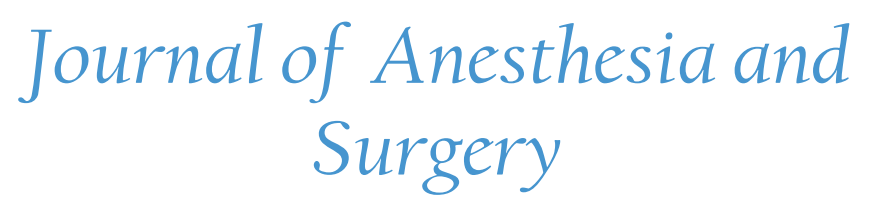

\title{
Prediction of Difficult Laryngoscopy in Pregnant Women Un- dergoing Cesarean Section Using the Hyomental Distance in fully extended and neutral position of neck, in Comparison With four usual bedside tests: A prospective blinded study
}

\author{
Anahita Hirmanpour ${ }^{1}$, Mohammadreza Safavi ${ }^{*}$, Azim Honarmand ${ }^{1}$, Seyyed Mohammad Moosavi ${ }^{1}$, \\ Shokooh Sadeghi ${ }^{1}$
}

${ }^{1}$ Department of Anesthesiology and Critical Care Research Center, Isfahan University of Medical Sciences, Isfahan, Iran

*Corresponding author: Mohammadreza Safavi, Professor, Department of Anesthesiology and Critical Care Research Center, Isfahan University of Medical Sciences, Isfahan, Iran, Tel: +989133152416, Fax: 00983112732659;

E-mail: safavi@med.mui.ac.ir

\begin{abstract}
Background: Unexpected difficult intubation that maybe considered to failed intubation is a major factor to be related to mortality and morbidity following general anesthesia. We aimed to elucidate the role of hyomental Distance in fully extended and neutral position of neck with other prevailing test and their possible correlation in predicting difficult laryngoscopy in parturient undergoing cesarean section.

Materials and Methods: After institutional approval and obtaining inform consents, 716 consecutive parturient ASA physical status I and II scheduled for elective cesarean section under general anesthesia, were enrolled to this study. Each patient was evaluated regarding Hyomental distance in extended (HMDe) and neutral position of neck(HMDn), Neck circumference to Thyromental Distance(NC/TMD), Ratio of height to Thyromental Distance(RHTMD), Modified Mallampatti Test (MMT) and Upper Lip Bite Test(ULBT) before induction. Laryngoscopic result was graded according to Cormack-Lehane Classification. Sensitivity, specificity, positive predictive value and AUC or ROC for each airway predictor in isolation and in comparison with each other was established.

Results: The sensitivity of HMDe and HMDn was 49.2 and $47.7 \%$ respectively. Sensitivity of MMT as an old predictive test was $79.3 \%$ in comparison with sensitivity of NC/TMD, RHTMD and ULBT (58.3\%, 41.6\% and 50.7\% respectively). The differences of Area under Curve in all tests except ULBT were statistically significant $(\mathrm{P}<0.05)$.

Conclusion: In addition to MMT (as an ancient predictor), NC/TMD and HMD in neutral position and fully extended of the neck; in parturient are good and reliable predictors of difficult laryngoscopy and intubation.
\end{abstract} Received date: April 4, 2016 Accepted date: June 11, 2016 Published date: June 15, 2016

Citation: Hirmanpour, A., et al. Prediction of Difficult Laryngoscopy in Pregnant Women Undergoing Cesarean Section Using the Hyomental Distance in fully extended and neutral position of neck, in Comparison With four usual bedside tests: A prospective blinded study. (2016) J Anesth Surg 3(1): 135-141.

DOI: $10.15436 / 2377-1364.16 .038$

Keywords: Caesarean section; General anesthesia; Laryngoscopy; Intubation

\section{Introduction}

The most responsibility of an anesthesiologist, especially in management of parturient, is to ability to predict difficult laryngoscopy and intubation and maintain the potency of ventilation and adequate gas exchange. Although performing of general anesthesia in obstetrics has significantly declined in recent centuries, but it is still inevitable in special situation, such as: Massive Maternal Hemorrhage, Overt Coagulopathy, Fetal Bradycardia which is life threatening and finally patient refusal for neuraxial 
techniques. The last reason mentioned above is one of the most indications of general anesthesia in obstetrics in developing countries.

Failure to achieve endotracheal intubation is a tangible endpoint that causes morbidity and mortality ${ }^{[1]}$. The incidence of failed intubation has been reported in a range of $0.7-31.3 \%{ }^{[2]}$. But in obstetric this has been considered in approximately ten times greater than general population ( 1 in 250). Most of the airway difficulties happen when they are not predict before anesthesia. So a skilled anesthesiologist should have the ability to determine the difficulties with airway management ${ }^{[3,4]}$.

Recently" obstetrics $^{[6]}$. Also RHTMD (Ratio of Height to Thyromental Distance) that has high sensitivity, is sufficiently sensitive to detect possible difficulties with laryngoscopy and intubation in obstetrics(AUC $=0.627,95 \% \mathrm{CI}, 0.589-0.664)^{[7]}$.

The modified Mallampati classification has poor prognostic value ${ }^{[2,6-8]}$ in many studies. In addition, Savva ${ }^{[8]}$ showed that modified Mallampati test (MMT) was neither sensitive nor specific enough as a single test in predicting difficult intubation in parturient patients.

In one study Honarmand et al. ${ }^{[9]}$ showed that HMDR (Hyomental Distance Ratio) is comparable with RHTMD and ULBT in prediction difficult airway. Also Takenaka et al. ${ }^{[10]}$ showed that HMDR is a clinically reliable predictor of DVL in general population.

These years, description of upper lip bite test (ULBT) by Khan et al. ${ }^{[11,12]}$ has become under scrutiny.

No published study has compared HMDR with ULBT, RHTMD, MMT, and NC/TMD by their sensitivity, specificity, and positive and negative predictive values for prediction of difficult laryngoscopy in pregnant patients.

Therefore, the hypothesis underlying this study was to develop predictors for difficult intubation in paturients candidate for cesarean section under general anesthesia and to test that HMDR has a positive correlation with other mentioned indices and which of them has a direct correlation with difficult laryngoscopic view and difficult intubation.

\section{Materials and Methods}

This prospective observational study was approved in our university ethics committee and all patients provided informed and written consent.

Patients with a history of trauma to the airway or cranial, cervical spine fracture, cervical and facial regions pathology, or were edentulous or requiring awake intubation, patients with restricted motility of the neck and mandible (e.g., cervical disc disorders or rheumatoid arthritis) or inability to sit were not included in the study.

During the $18^{\text {th }}$ month period, 716 consecutive American Society of Anesthesiologists (ASA) physical status I and II adult patients who were undergoing elective cesarean delivery under general anesthesia with tracheal intubation were enrolled into this prospective observational study.

The subsequent five measurements of predictive test were performed in all patients:

HMDR: The ratio of hyomental distance in full extension of neck to this distance in neutral position. (As shown in Figure 1) ${ }^{[10]}$.

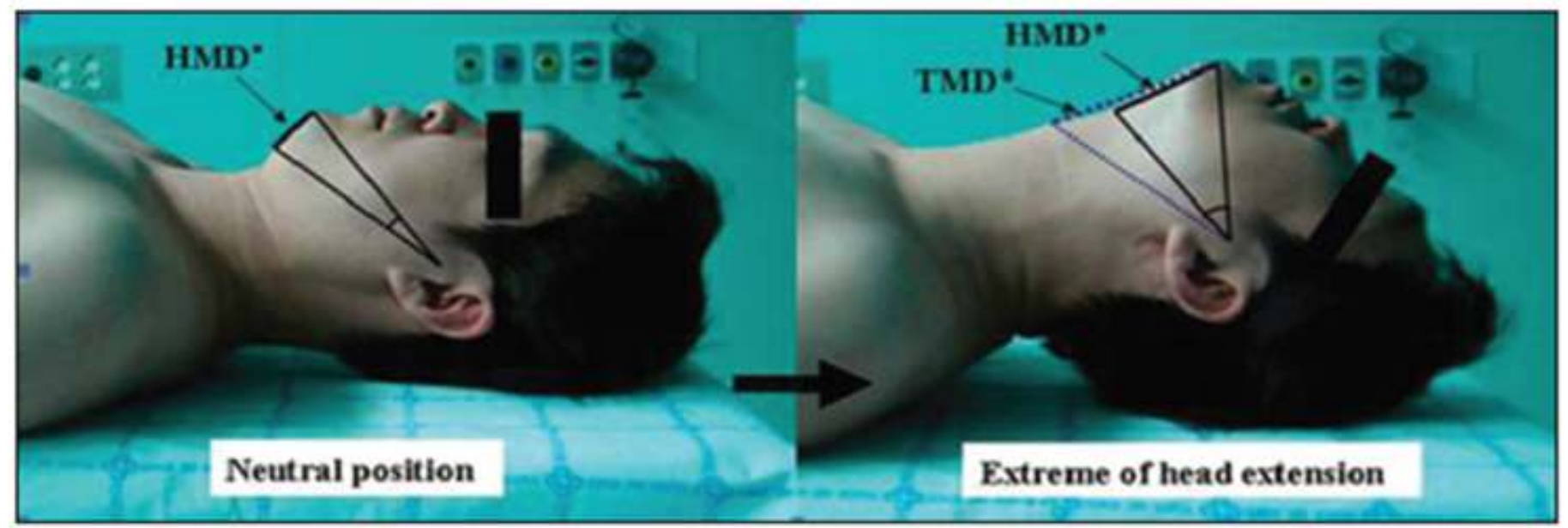

Figure 1: Method for measuring the hyomental distance ration. The HMDR was defined as the ration of the hyomental distance at the extreme of head extension (expressed as HMDe) to that in the neutral position (expressed as HMDn). Thyromental distance at the extreme of head extension was expressed as TMD.

NC/TMD: The neck was measured at the level of cricoid cartilage and thyromental distance was measured from the bony point of the mentum while the head was fully extended with close mouth. The ratio was calculated ${ }^{[5]}$.

RHTMD: Thyromental distance was measured from the bony point of the mentum while the head was fully extended with close mouth. Then the ratio of height to TMD was calculated ${ }^{[7]}$.

MMT: Modified Mallampati classification as described by Samsoon and Young. Classes are differentiated on the basis of the struc- 
tures visualized: class I:soft palate, fauces, uvula, tonsillar pillars; class II: soft palate, fauces, uvula; class III: soft palate, base of the uvula; class IV: soft palate not visible.

ULBT: ULBT was introduced as follows: Class I: The lower incisors can bite the upper lip above the vermilion line; Class II: Lower incisors could bite the upper lip below the vermilion line; Class III: Lower incisors could not bite the upper lip ${ }^{[11]}$.

Each parturient data was collected by an anesthetic nurse included age, weight, height and body mass index (BMI) and the airway parameters. A skilled anesthesiologist with at least 5 years experience in anesthesia not imparted of the noted preoperative airway assessment, carried out laryngoscopy and rating the difficulty of intubation ${ }^{[13,14]}$. The Cormack-Lehane grading system for laryngoscopic view is defined: Grade 1 is visualization of the entire laryngeal aperture; grade 2 visualization of only the posterior portion of the laryngeal aperture; grade 3 is visualization of only the epiglottis; and grade 4 is no visualization of the epiglottis or larynx. Difficult visualization of larynx (DVL) was defined as grade 3 and 4 of laryngoscopy.

Each parturient who received the aspiration prophylaxis ${ }^{[15]}$, preoxygenated for five minutes and anesthesia was induced intravenously with sodium thiopental $(5 \mathrm{mg} / \mathrm{kg})$ and suxamethonium chloride $(2 \mathrm{mg} / \mathrm{kg})$ for facilitating endotracheal intubation. Sellick maneuver ${ }^{[16]}$ was applied until the patient was intubated and the cuff was inflated and confirmed successful intubation by bilateral auscultation of lungs and capnography. For the first laryngoscopy in each case, size 3 of Macintosh laryngoscope blade was used $^{[17]}$.

Difficult visualization of larynx (DVL) has been defined the grade 3 and 4 in classification of Cormack and Lehane, and grade 1 and 2 of this classification are defined as easy visualization of larynx(EVL).

All these clinical data (the Mallampati score, the ULBT score, HMDe, HMDn, NC/TMD and RHTMD) were recorded for each patient. Sensitivity, specificity, Positive Likelihood ratio(+LR) and Negative Likelihood ratio(-LR), positive and negative predictive value(PPV,NPV respectively) of each test were calculated.

The AUC was used as the main end-point of the study to decide whether the score was clinically useful. A value of 0.5 under the ROC curve indicated that the variable performs no better than chance and a value of 1.0 indicates perfect discrimination. The ROC curve was used to determine the optimal predictive cutoff point for each test and a larger area under the ROC curve represents more reliability ${ }^{[18,19]}$ and good discrimination of the scoring system.

$\mathrm{P}$ value of 0.05 was defined as statistically significant. Differences between the AUC values of the predictor tests were analyzed using Med Calc statistical software 9.3.6.0. The other calculations were analyzed using SPSS version 20.

\section{Sample Size Selection}

A prospective power analysis revealed that assuming an incidence of difficult laryngoscopy of $15 \%, 761$ Parturient provide a power of more than $80 \%$ to detect an improvement of discriminating power (measured by the area under Receiver operating characteristic (ROC) curve) of an absolute value of 7\% with a type I error of 5\% and using a two-sided alternative hypothesis.

Patient data were presented as mean \pm SD BMI and value of the airway predictors were compared using t-tests for continuous variables and U-test for MMT or ULBT. Sensitivity, specificity, and Positive predictive value PPV were obtained and compared amongst predictors.

\section{Results}

A total of 716 patients were enrolled into this study. We have 3 cases with grade IV CL. The tracheal intubations in these patients were performed with video laryngoscope. All demographic data such as patient's age, height, weight and BMI are shown in Table 1. There are no significant differences in demographic data between EVL and DVL.

Table 1: Patients characteristics

\begin{tabular}{|l|l|l|l|l|}
\hline Variables & Patients $(\mathbf{n}=\mathbf{7 1 6})$ & EVL(n= 584) & DVL(n= 132) & Pvalue \\
\hline Age(years) & $28.8 \pm 4.9$ & $28.7 \pm 4.9$ & $29.1 \pm 4.9$ & 0.428 \\
\hline Weight(kg) & $76.7 \pm 12$ & $76.5 \pm 12$ & $77.4 \pm 12.1$ & 0.440 \\
\hline Height(cm) & $161 \pm 6.1$ & $161 \pm 6.3$ & $160 \pm 5.6$ & 0.082 \\
\hline BMI $\left(\mathbf{k g} / \mathbf{m}^{\mathbf{2}}\right)$ & $29.6 \pm 4.3$ & $29.5 \pm 4.3$ & $29.8 \pm 4.2$ & 0.398 \\
\hline
\end{tabular}

EVL: Easy visualization of larynx, DVL: Difficult visualization of larynx, BMI: Body mass index, Data are presented as mean \pm SD. $\mathrm{P}<0.05$ statistically significant. 
Prediction of Difficult Laryngoscopy in Cesarean Section

Table 2: Distribution of ASA, MMT, ULBT and laryngoscopic view of all patients

\begin{tabular}{|l|c|}
\hline Variable & Number(\%) \\
\hline ASA & $573(80)$ \\
\hline I & $143(20)$ \\
\hline Mallampati Class & $293(40.9)$ \\
\hline I & $263(36.7)$ \\
\hline II & $124(17.3)$ \\
\hline III & $36(5)$ \\
\hline IV & \\
\hline ULBT & $390(54.5)$ \\
\hline I & $320(44.7)$ \\
\hline II & $6(0.8)$ \\
\hline III & \\
\hline Laryngoscopic view & $362(50.6)$ \\
\hline I & $222(31)$ \\
\hline II & $129(18)$ \\
\hline III & $3(4)$ \\
\hline IV & \\
\hline
\end{tabular}

ASA: American Society of Anesthesiology, ULBT: Upper Lip Bite Test

The distribution of ASA, MMT, ULBT and the Cormack- Lehane grading are presented in Table 2.

Table 3 is shown that the differences on NC/TMD, HMDe and HMDn in statistically significant in comparison with the other tests.

Table 3: Distribution of Statistically differences in all tests in DVL and EVL

\begin{tabular}{|l|r|r|c|}
\hline Variables & DVL(n = 132) & EVL(n= 584) & P value \\
\hline TMD & $8.9 \pm 1.5$ & $9.2 \pm 1.4$ & 0.025 \\
\hline RHTMD & $18.5 \pm 3.6$ & $17.8 \pm 2.9$ & 0.010 \\
\hline NC & $36.5 \pm 2.5$ & $35.9 \pm 2.8$ & 0.018 \\
\hline NC/TMD & $4.2 \pm 0.8$ & $3.9 \pm 0.6$ & 0.000 \\
\hline HMDe & $6.6 \pm 1$ & $7.2 \pm 1$ & 0.000 \\
\hline HMDn & $4.9 \pm 1.1$ & $5.4 \pm 1.1$ & 0.000 \\
\hline HMDR & $1.38 \pm 0.2$ & $1.37 \pm 0.2$ & 0.497 \\
\hline
\end{tabular}

DVL: Difficult view of laryngoscopy, EVL: Easy view of laryngoscopy, TMD: Thyromental distance ratio, RHTMD: Ratio of height to thyromental, NC: Neck circumference, NC/TMD: Neck circumference to thyromental distance, HMDe: hyomental distance in extention of neck, HMDn: Hyomental distance in neutral position of neck, HMDR: ratio of HMDe/HMDn. Data is presented as mean $\pm \mathrm{SD}$. P value $<0.05$ is significant.

The predictive value of MMT, ULBT, NC/TMD, RHTMD, HMDe, HMDn, HMDR are presented in Table 4. The main end point in this study, the AUC of the ROC, were lower for ULBT (AUC $=0.532,95 \% \mathrm{CI}=0.494-0.569)$ and HMDR $(\mathrm{AUC}=0.551$, $95 \% \mathrm{CI}=0.514-0.588)$ and RHTMD $(\mathrm{AUC}=0.555,95 \% \mathrm{CI}=0.517-0.591)$ in comparison with $\mathrm{MMT}(\mathrm{AUC}=0.582,95 \% \mathrm{CI}$ $=0.545-0.619), \mathrm{NC} / \mathrm{TMD}(\mathrm{AUC}=0.600,95 \% \mathrm{CI}=0.563-0.637), \mathrm{HMDe}(\mathrm{AUC}=0.672,95 \% \mathrm{CI}=0.636$ to 0.706$)$ and $\mathrm{HMDn}$ $(\mathrm{AUC}=0.651,95 \% \mathrm{CI}=0.614$ to 0.686$)$. Figures 2-7. The differences of the last four ROCs were statistically significant $(\mathrm{P}<0.05)$.

Table 4: Predictive value for MMT,ULBT, RHTMD,NC/TMD, HMDe, HMDn and HMDR to predict the occurrence of DVL according to the modified Cormack- Lehane Classification

\begin{tabular}{|l|c|c|c|c|c|c|c|c|c|c|}
\hline Test & Sensitivity & $\mathbf{9 5 \% C I}$ & Specifisity & $\mathbf{9 5 \%}$ CI & +LR & -LR & PPV (\%) & NPV (\%) & AUC of ROC curve & P value \\
\hline MMT & 73.4 & $65.1-80.8$ & 44.1 & $40.1-48.3$ & 1.32 & 0.6 & 22.9 & 88.1 & 0.582 & 0.003 \\
\hline UBLT & 50.7 & $41.9-59.6$ & 55.6 & $51.5-59$ & 1.14 & 0.88 & 20.6 & 88.3 & 0.532 & 0.262 \\
\hline RHTMD & 41.6 & $33.2-50.6$ & 75 & $71.3-78.1$ & 1.67 & 0.78 & 27.4 & 85 & 0.555 & 0.053 \\
\hline NC/TMD & 58.3 & $49.4-66.8$ & 64.3 & $60.3-68.3$ & 1.64 & 0.65 & 27 & 87.2 & 0.600 & 0.000 \\
\hline HMDe & 49.2 & $40.4-58.1$ & 79.2 & $75.8-82.5$ & 2.38 & 0.64 & 34.9 & 87.4 & 0.672 & 0.000 \\
\hline HMDn & 47.7 & $39-56.5$ & 82.8 & $79.5-85.8$ & 2.78 & 0.63 & 38 & 87.5 & 0.651 & 0.000 \\
\hline HMDR & 45.4 & $36.8-54.3$ & 73.4 & $69.6-77$ & 1.71 & 0.74 & 27.9 & 85.6 & 0.551 & 0.071 \\
\hline
\end{tabular}


MMT: Modified Mallampati Test, ULBT: Upper lip bite test, RHTMD: Ratio of height to thyromental distance, NC/TMD: Ratio of neck circumferences to thyromental distance,HMDe: hyometal distance in head fully extended with closed mouth, HMDn: hyomental distance in neutral position, HMDR: hyomental distance ratio, CI: Confidence Interval, AUC: Area under curve, ROC: Receiver- operating characteristic curve. P $<$ 0.05 statistically significant.

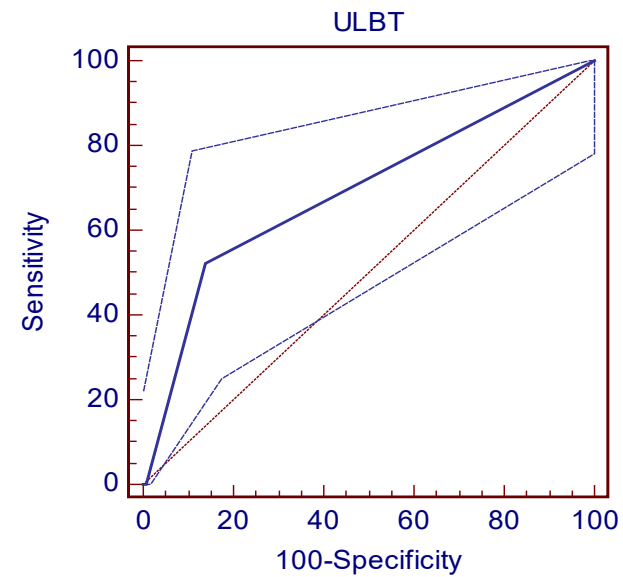

Figure 2: AUC of ROC of Upper Lip Bite Test

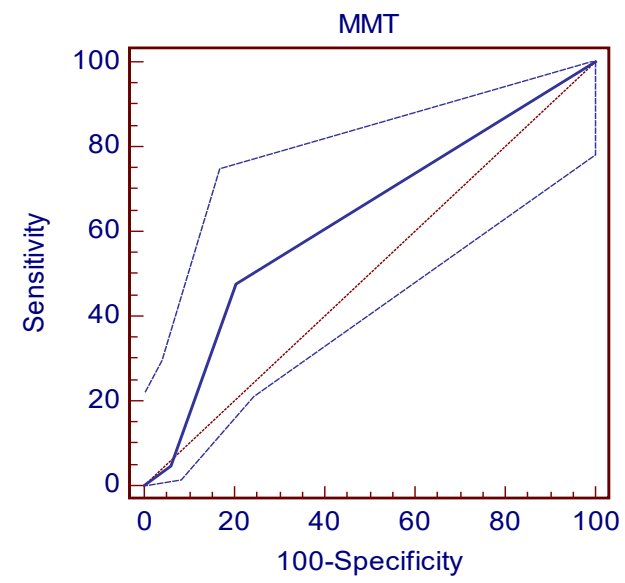

Figure 4: AUC of ROC of Modified Mallampati test

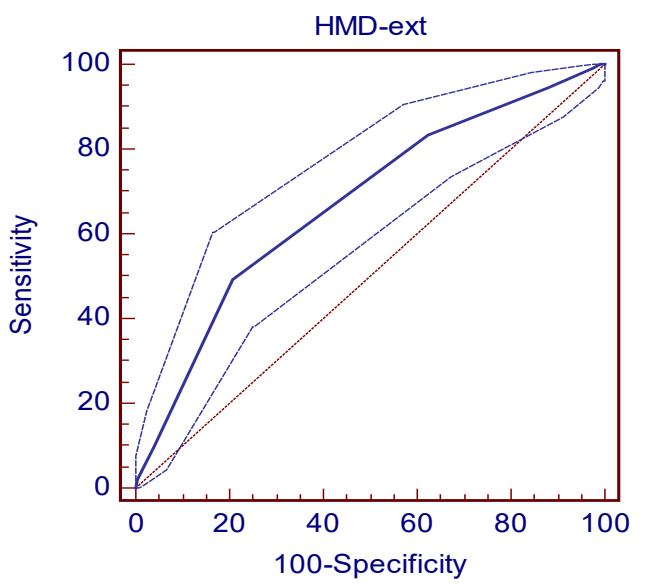



Figure 3: AUC of ROC of Ratio of Height to Thyromental distance

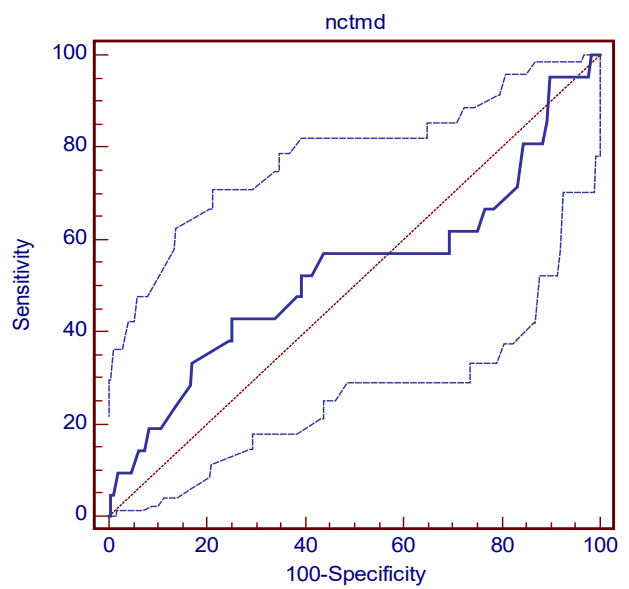

Figure 5: AUC of ROC of Neck Circumference to Thyromental

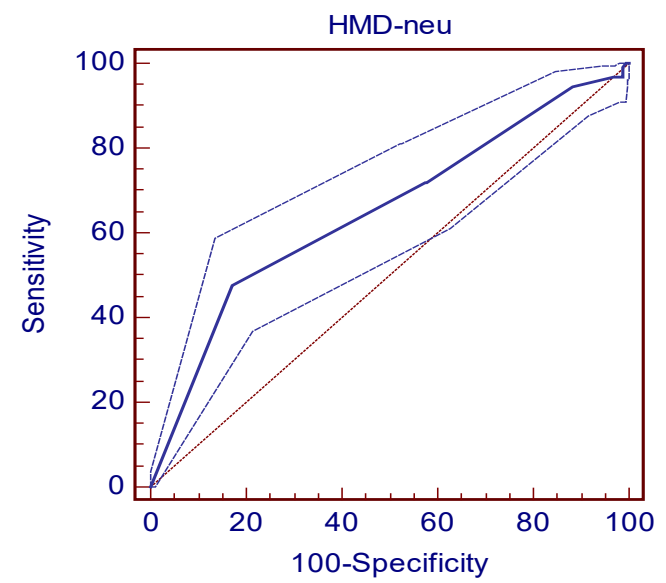

Figure 6: AUC of ROC of Hyomental Distance in full extention of neck Figure 7: AUC of ROC of Hyomental Distance in neutral position Distance of neck

In discrimination analysis, MMT grade $>$ I, ULBT grade $\geq$ II, RHTMD $\geq 19.2$, NC/TMD $\geq 4.1, \operatorname{HMDe} \leq 6, \mathrm{HMDn} \leq 4$ and HMDR $\geq 1.4$ were considered as the cutoff point in predicting DVL. The RHTMD is the least sensitive tests with the sensitivity of 41.6\%. MMT and NC/TMD had the highest sensitivities among the predictors ( $73.4 \%$ and 58.3 respectively). 
Discussion

Difficult laryngoscopy and intubation can cause irreparable sequel for the patient if not handled properly. Studies to find predictive tests with high accuracy continue. Little works have been published based on the use HMDR( HMDe/HMDn), MMT, ULBT, RHTMD and NC/TMD in obstetrics airway management. This study was designed to evaluate the efficacy of the five tests above in forecasting difficult laryngoscopy and to show a possible correlation between the tests and Cormack-Lehane grade of laryngoscopy. The previous studies have shown the incidence of difficult intubation $1.3-17 \% \%^{[2,20]}$ and it is ten times more in parturient $(13-170 \%)^{[6]}$. In this study, the incidence of DVL was $18.4 \%$ that was comparable with previous studies. In Merah et al. ${ }^{[21]}$ study, the incidence of DVL in Niegerian parturients was $10 \%$. We can support our finding by presence some differences in head position, degree of muscle relaxation and different anthropometric features.

Some studies have shown increasing weight and BMI as a risk factor for DVL ${ }^{[22]}$, yet others ${ }^{[23,24]}$ and the present study in parturient have found that the incidence of DVL was not correlated with BMI. It may be due to not using general anesthesia in these parturient candidates for elective cesarean section.

In this study ULBT failed to be as a bedside test in predicting DVL. The descriptive reason was the little amount of the cases with ULBT grade III $(0.8 \%)$ and only $0.1 \%$ had DVL. On the other hand in patients who had ULBT I and II the probability of DVL was exceeding low which is comparable with previous studies ${ }^{[11,12]}$.

The accuracy of NC and NC/TMD for prediction of DVL in non obstetric patients was documented by Gonzalez ${ }^{[25]}$ and Kim et al. ${ }^{[15]}$. In this study and the previous one ${ }^{[6]}$, we found that $\mathrm{NC}$ and NC/TMD was the most useful predictors in parturient with AUC of ROC $0.564(\mathrm{P}=0.022)$ and $0.600(\mathrm{P}=0.000)$ respectively.

The $73.4 \%$ sensitivity of the mallampati test combined with $22.9 \%$ positive predictive value, suggests that for a reliable prediction of difficult laryngoscopy, Mallampati scoring should be combine with other predictors, however in this study the AUC of ROC of Mallampati test is $0.582(\mathrm{P}=0.003)$.

Hyomental distance( measured in supine position with the head fully extended and with the mouth closed as the straight distance from the lower border of the mandibular mentum to the superior border of the hyoid bone in centimeters) that was described HMDe in this study, had a significant difference in DVL $(\mathrm{P}<0.001)$. Also HMDn (measured in supine position with head in neutral position) is a good predictor of difficult intubation $(\mathrm{P}<0.001)$. In khan et al. ${ }^{[12]}$ study described the HMD is a valuable predictor of DVL. But HMDR has the sensitivity of $45.4 \%$ and is not a good predictor in the parturient.

In conclusion, there are stepwise increases in the incidence of Cormack-Lehane grade III and IV as the MMT class shows a rise form II to III and more, NC/TMD increases from the value of 4.1, HMDe and HMDn decreases from their predetermined value of 6 and $4 \mathrm{~cm}$, respectively. So we consider neck circumference to thyromental distance and hyomental distance in neutral position and fully extended neck with closed mouth are good predictors of difficult laryngoscopy and intubation in obstetric patients.

Our study demonstrated that in addition to MMT ( as an ancient predictor), NC/TMD and HMD in neutral position and fully extended of the neck, in parturient with higher incidence of DVL rather than general population, are good and reliable predictors of difficult laryngoscopy and intubation using a standard laryngoscope.

\section{APPENDIX}

\section{STATISTICAL TERMS AND DEFINITIONS}

- True positive: A difficult laryngoscopy that had been predicted to be difficult (A)

- False positive: An easy laryngoscopy that had been predicted to be difficult (B)

- True negative: An easy laryngoscopy that had been predicted to be easy (D)

- False negative: A difficult laryngoscopy that had been predicted to be easy (C)

- Sensitivity: The percentage of correctly predicted difficult laryngoscopies as a proportion of all laryngoscopies that were truly difficult (A/(A +

C))

- Specificity: The percentage of correctly predicted easy laryngoscopies as a proportion of all laryngoscopies that were truly easy $(\mathrm{D} /(\mathrm{B}+\mathrm{D})$

- Positive predictive value: The percentage of correctly predicted difficult laryngoscopies as a

proportion of all predicted difficult laryngoscopies $(\mathrm{A} /(\mathrm{A}+\mathrm{B}))$

- Negative predictive value: The percentage of correctly predicted easy laryngoscopies as a

proportion of all predicted easy laryngoscopies $(D /(C+D))$

- Accuracy: The percentage of correctly predicted easy or difficult laryngoscopies as a proportion of all laryngoscopies $(\mathrm{A}+\mathrm{D}) /(\mathrm{A}+\mathrm{B}+\mathrm{C}+\mathrm{D})$

- Likelihood ratio of a positive test result (LR+): The number of times more likely that a patient with positive test result will have a difficult airway; it is calculated by sensitivity divided by 1 -specificity.'

- AUC of an ROC-curve (area under a receiver operating characteristic curve): The probability of the correct classification using the test in a sample pair of two patients (one with an easy airway, one with a difficult airway). In this specialized case the AUC= (sensitivity* $(1$-specificity $) / 2)+$ (sensitivity* specificity) $+\left((1 \text {-sensitivity })^{*}\right.$ specificity/2) 


\section{References:}

1. Cooper, G.M., McClure, J.H. Anesthesia chapter from Saving mothres' lives; reviewing maternal deaths to make pregnancy safer. (2008) Br J Anaesth 100(1): 17-22.

2. Londstrom, L.H., Vester-Anderson, M., Moller, A.M., et al. Poor prognostic value of the modified Mallampati score: a meta-analysis involving 177088 patients. (2011) Br J Anaesth 107(5): 659-667.

3. Khan, Z.H. Airway Assessment: A Critical Appraisal. (2014) Airway management 15-32.

4. Palanisamy, A., Matni, A.A., Tsen, L.C. General anesthesia for cesarean delivery at a tertiary care hospital fron 2000-2005: A retrospective analysis and 10-years update. (2011) Int J Obstet Aneasth 20(1): 6-10.

5. Kim, W.H., Ahn, H.J., Lee, C.J., et al. Neck circumference to thyromental distance ratio: A new predictor of difficult intubation in obese patients. (2011) Br J Anaesth 106(5): 743-748.

6. Hirmanpour, A., Safavi, M., Honarmand, A., et al. The predictive value of the ratio of neck circumference to thyromental distance in comparison with four predictive tests for difficult laryngoscopy in obstetric patients scheduled for cesarean delivery. (2014) Adv biomed Res 3: 200.

7. Honarmand, A., Safavi, M., Zare, N., A comparison of the ratio of patient's height to thyromental distance with the modified Mallampati and the upper lip bite test in predicting difficult laryngoscopy. (2011) Saudi J Anaesth 5(3): 258-263.

8. Savva, D. Prediction of difficult tracheal intubation. (1994) Br J Anaeth 73(2): 149-153.

9. Honarmand , A., Safavi, M.R., Ansari, N. A comparison between hyomental distance ratio, ratio of height to thyromental, modified mallampati classification test and upper lip bite test in predicting difficult laryngoscopy of patients undergoing general anesthesia. (2014) Adv Biomed Res 3: 166.

10. Takenaka, I., Iwagaki, T, Aoyama, K., et al. Preoperative evaluation of extension capacity of the occipotoatlantoaxial complex in patient with Rheumatoid Arthritis: Comparison between the Bellhouse test and a new method, hyomental distance ratio. (2006) Anesthesiology 104(4): 680685 .

11. Khan, Z.H., Kashfi, A., Ebrahimkhani, E. A comparison of the upper lip bite test (a simple new technique) with modified Mallampati classification in predicting difficulty in endotracheal intubation: A Prospective blinded study. (2003) Anesth Analg 96(2): 595-599.

12. Khan, Z.H., Maleki, A., Makarem, J., et al. A comparison of the upper lip bite test with hyomental/thyrosternal distances and mandible length in predicting difficulty in intubation. (2011) Indian J Anaesth 55(1): 43-46.

13. Williams, K.N., Carli, F., Cormack, R.S. Unexpected difficult laryngoscopy: a prospective survey in routine general surgery. (1991) Br J Anaesth 66(1): 38-44.

14. Krage, R., van Rijn, C., van Groeningen, D., et al. Cormack-Lehane classification revisited. Br J Anaesth 105(2): 220-227.

15. Mallampati, S.R. Recognition of the difficult airway. In Benumof JL, editor: Airway management principles and practice. (1996) St Louis Mosby 132.

16. American Society of Anesthesiologists Task Force on Obstetric Anesthesia. Practice guidelines for obstetric anesthesia: an updated report by the, American Society of Anesthesiologists Task Force on Obstetric Anesthesia. ( 2007) Anesthesiology 106(4): 843-863

17. Sellick, B.A. Cricoid pressure to control regurgitation of stomach contents during induction of anesthesia. (1961) Lancet 2(7199): 404-406

18. Schniederjans, M.J. Mathematical partitioning of the receiver operating curve: A diagnostic tool for medical decision making. (1985) Socio-Economic Planning Sciences 19(2):125-135.

19. Hanley, J.A., McNeil, B.J. The meaning and use of the area under a receiver operating characteristic (ROC) curve. (1982) Radiology 143(1): 29-36.

20. Gupta, S., Sharma, K.R., Jain, D. Airway assessment: Predictors of difficult intubation. (2005) Indian J Anesth 49(4): $257-262$.

21. Merah, N.A., Foullkess-Crabbe, D.J., Kushimo, O.T., et al. Predictors of difficult laryngoscopy in population of Nigerian Obstetric patients. (2004) West Afr J Med 23(1): 38-41.

22. Prasada Rao, D., Rao, V.A. Morbidly obese parturient: Challenges for the anaesthesiologist, including managing the difficult airway in obstetrics. What is new?. (2010) Indian J Aneasth 54(6): 508-521.

23. Brodsky, J.B., Lemmens, H.J., Brock-Utne, J.G., et al. Anaesthetic considerations for bariatric surgery. Proper positioning is important for laryngoscopy. (2003) Anaesth Analg 96(6): 1841-1842.

24. Bond, A. Obesity and difficult intubation. (1993) Aneasth Intensive Care 21: 828-830.

25. Gonzales, H., Minville, V., Mazerolles, M., et al. The importance of increase neck circumference to intubation difficulties in obese patients. (2008) Anesth Analg 106(4): 1132-1136.

Ommega Online Publishers

Journal Title: Journal of Anesthesia and Surgery (JAS)

Journal Short Name: J Anesth Surg
Journal ISSN: 2377-1364

E-mail: anestheisa@ommegaonline.com

Website: www.ommegaonline.org 